\title{
Téoros
}

Revue de recherche en tourisme

\section{L'apport ethnique dans le tourisme des Laurentides}

\section{Christian Morissonneau}

Volume 15, numéro 1, printemps 1996

Les Laurentides, quelles Laurentides?

URI : https://id.erudit.org/iderudit/1075050ar

DOI : https://doi.org/10.7202/1075050ar

Aller au sommaire du numéro

Éditeur(s)

Université du Québec à Montréal

ISSN

0712-8657 (imprimé)

1923-2705 (numérique)

Découvrir la revue

Citer cet article

Morissonneau, C. (1996). L'apport ethnique dans le tourisme des Laurentides. Téoros, 15(1), 26-29. https://doi.org/10.7202/1075050ar d'utilisation que vous pouvez consulter en ligne.

https://apropos.erudit.org/fr/usagers/politique-dutilisation/ 


\section{LAPPORT E'THNTQUE DANS LE TOURISME DES LAURENTIDES}

\section{Christian Morissonneau}

\author{
Christian Morissonneau \\ Département \\ des Sciences Humaines \\ Université du Québec \\ à Trois-Rivières
}

Cet article ne prétend pas faire la description exhaustive des représentants des communautés culturelles dans le développement touristique laurentidien. II présente des données qul auralent besoln d'être aftlnées entre autres par le dénombrement, aussı par l'étude des motlvations, de l'accuell des résidants et autres touristes québécols, des rapports inter-ethnlques, de l'apport économlque et soclo-culturel, de l'entrepreneurship, etc., qui demeurent des champs d'analyse blen mal connus sinon inconnus (beaucoup de mémolres et de thèses possibles!). Que le lecteur y vole d'abord une introduction à ces études et surtout une invitation. Et puls, vollà que les études ethnlques devlennent un des domaines où le para-sclentiflque donne le ton! Les solltudes vont durer encore longtemps...

\section{Aux origines, la villégiature}

L'ensemble géographlque des Laurentldes (réglonalement tradult par les réglons des Laurentides et de Lanaudière) n'attire pas seulement, depuls les débuts de la villégiature, que les Canadlens-Françals catholiques. En beaucoup d'endrolts, les anglophones et les représentants des autres ethnles ont lancé les premlers des fronts plonnlers touristiques dans les sites attrayants des nappes d'eau et des montagnes. Ces villéglateurs ont contribué à la construction des régions. Les Laurentides en sont le mellleur exemple.

Il nous faut commencer par la description de ce mouvement. Si je pose que Terrebonne est une des portes des Laurentides, nous voyons la villéglature s'amorcer dès la fin du XVIIIe slècle, le long de la rivlère des Mille Iles qul devlent ainsI le premier Nord touristique de Montréal. Des représentants de la compagnle du Nord-Ouest. Mc Kenzle et Mc Tavish, font partle du tout premler groupe villéglaturant de Terrebonne. Et comme II s'aglt de leaders, d'autres les Imiteront avec leur résidences d'êté cossues.

Au début de notre slècle. *...quelques Anglals possèdent des résidences au pled du Grand Coteau, et au-dessus de lul. dans les sables, les Ternebonne Helghts sont plquetées de petits cottages britanniques et julfs $[\ldots], 1, \ldots]$ ces résidences (malsons de campagne) se font de plus en plus nombreuses [...l (le long des Mille lles) à Saint-Maurlce, surtout françals, à Rosemere, plutôt britannlque: 3 ooo tourlstes fréquentent la paroissem. (Blanchard, 1953:164).

A SaInt-Lin, on compte quelques familles dont certaines julves $₫ / .$. J dans les malsons devenues llbres après l'ém fgration causée par la lièvre du foln: c'est l'avant-garde des colonles fulves de New-Glasgow et SainteSophle-de-Lacorne* (Blanchard, 1953:162)

Ainsı, autant pour les francophones que les anglophones, le tourisme est né dans les Basses-Laurentides qui conserveront leur prépondérance face aux Hautes-Laurentıdes, au molns jusque dans les années 1920. Mals déjà des représentants des ethnles de Montréal et d'allleurs particlpalent au mouvement touristique plus au Nord.

\section{Les clubs de chasse et pêche}

La dernlère pelletée de terre sur la route de Saint-Jérôme à Sainte-Agathe-des-Monts, en 1856, falt assez de brult qu'elle annonce le grand sbond en avant* touristique. Les clubs privés organisent des expédittons de chasse et de pêche pour les notables. Ceux-cl deviennent autant de leaders d'opinlon qul donnent la notorlété *plein alr* de cette région à pelne ouverte. Le tourisme seralt-Il le principal prodult attractuf? Le rêve du curé Labelle de faire du Nord *une grande Sulsse canadienne" allalt-ll se réalliser? Les villages se disputent aussı blen les Canadiens-Françals que les *étrangers*. Salnte-Adèle, qui ne connaít pas son avenir, envle SalnteAgathe-des-Monts pour cette Installation des riches cosmopolites. sainte-Agathe devlent en peu d'années le centre de pleln alr de prédllection du gratın, d'une bourgeolsle canadlenne, amérlcalne et européenne qul s"Installe autour du lac des Sables: le rol du sucre Baumgarten, le minlstre Nantel, l'Industriel et courtier John Hendt, le vlcomte Raoul d'Ogler d'lvry... y ont de riches villas*. (Cité dans Laurin, 1989:362)

A tout le moins, vollà un endroit cosmopolite. La comtesse Ogier D'Ivry, qui est née au Mans en France, achète en 1891 la ferme du lac Manitou pour son fills Raoul dont elle souhaite l'établissement. C'est le patronyme que retlendra la municipalité qui se fonde trente ans plus tard. Un toponyme est alnsı né du nom de ce *vllléglateur permanent. La *ferme" devlendra un "chăteau* et le slège du club privé Manitou. Et Ivry-sur-le-Lac, avec ses riches chalets est surnommé le Westmount du Nord de Montréal... Du quartler ethnique au centre de villéglature!

Les plus riches des touristes sont plutôt membres des clubs privés. Ceux-cl attirent de nombreux millionnaires amérlcains. Les plus connus pour les fortunés anglophones: le club Chapleau dans les Laurentides et le club Mastigouche dans Lanaudière, qu'on surnomme *le club des millionnaires* (américains) et qu'on dit le plus riche du Québec dans la première moitté du slècle. Le club Chapleau fondé en 1895. avec quelques notables francophones, est fréquenté par des capitallstes américains; aussi par les Shaughnessy et Spencer du Canadien Pacilique. 


\section{Investissements et vogue du ski}

Au début du XXe slècle, une zone nouvelle s'ouvre au récréotourisme: les Pays-d'en-Haut, de Shawbridge au mont Tremblant. Le pmolongement du chemin de fer favorlsalt les déplacements vers le plein alr des hauts sommets et des nombreux lacs. Malgré l'énorme et raplde succès du ski, Il y aura un flottement dans l'achalandage: la vllléglature des Basses-Laurentides (et du Pledmont dans Lanaudière) attirent davantage de touristes, mals durant l'été, bien évidemment. Lanaudière ne fail pas partie de la zone touchée par la vague des skleurs à partir des années 1920. $\mathrm{m}$ Is à part Salnt-Gabrlel-de-Brandon, pourtant assez peu accidenté. Le développernent lanaudols du ski, sl on excepte quelques pentes peu spectaculaires, est récent. II connaît le succès depuls une quinzalne d'années à Val-Saint-Côme et n'est pas llé à l'apport ethnique, que ce solt pour l'initiative ou pour l'achalandage.

La vogue du skI dans les Laurentides n'est, pas venue d'abord d'une pratique canadienne-françalse. On va le volr.

Une date: 1905, où des membres du Montreal Ski Club, qui se contentent Jusque là des pentes du Mont Royal, partent en train pour Salnte-Agathe-des-Monts et descendent, sur leurs skls primitufs, vers Shawbridge dans une expédition qu'lls répètent pendant quelques années. A partir de 1911, une sorte de ski de fond va être organlsé par Émille Cochand, un instructeur originalre de Sulsse. II donne des cours et trace les premlères plstes à partir de l'auberge de SainteMarguerite-Station. Les membres du Montreal Ski Club organisent à Shawbridge les premlères compétitions. Ce petut village devient rapidement le berceau du ski régional.

Dans les années 1920. Sa inte-Agathe-des-Monts prend la tête des aménagements et attire de plus en plus d'amateurs. En 1928, les compétitions de skl retlennent une clientèle variée et font du village *le rendez-vous des sports des villes canadlennes et américalnes*. (LAvenir du Nord, 2 février 1928). Percy Douglas, celul qul a regroupé les clubs de ski de Montréal, de Trols-Rivières et d'Ottawa dans la Canadian Amateur Ski Association, pousse le CN à préparer des trallns spéclaux pour les skleurs montréalals.

\section{Ski de randonnée et ski alpin}

Les premlers trains de nelge en Amérique démarrent durant l'hlver 1927. Le Canadien Pacifique, organise aussitôt ses propres trains de nelge. II transporte, dès cette premlère salson, 11000 skleurs. Les clubs de ski rappellent les associations de colonisateurs des décennies précédentes: *Devlennent les plonnlers de I"Industrie tourlstuque, les Percy Douglas, Charles Bosvlk et surtout Herman *Jackrabblt Sm Ith Johannsen; IIs fondent des clubs et leur talent d'organIsateurs les propulse dans la nouvelle élite réglonale, composée de personnes étrangères à la réglon et souvent au pays* (Flllon. 1981). Ce sont effectivement, pour la plupart. des personnes d'origine étrangère.

L'hôtellerie de luxe avalt été lancée par des Anglo-Saxons, comme le Laurentide Inn à Sainte-Agathe-des-Monts ou le Gray Rocks Inn à Saint-Jovite. Cette dernlère auberge est ouverte par un Américain, George Ernest Wheeler. Celui-ci, né dans l'État de New York, venu pour un voyage de chasse, avalt amené plus tard sa famille, séduit par l'environnement, et s'était installé sur les rives du lac Oulmet. Au début du slècle II lance l'auberge qui devlendra célèbre. Ses fils poursulvent l'entreprise. On les revolt dans un autre grand projet.

Le grand plonnler du skl dans les Laurentides et au Canada demeure le Norvéglen, Herman Smith Johannsen, mort plus que centenaire, (1875-1987). En 1928, Il organlse pour les membres du Red Birds Skl Club, le premler slalom canadien. Des vrales pistes sont aménagées au mont Tremblant; elles permettent en 1931, d'organiser la premlère descente Taschereau, la plus ancienne course annuelle au Canada. Autre innovation américaine, cette fols à Shawbridge, un certain Foster installe le premier remonte-pente mécanlque en Amérique du Nord. Constrult avec un moteur Chrysler, on l'appelle le «Foster's follys, mals Il fonctionne... et les skleurs sont emballés. Un autre américain. Fred Pabst installe le premier remonte-pente permanent à Saint-Sauveur-desMonts en 1934. Ces apparells équipent très vite les stations. Les skieurs étrangers et Indlgènes ne peuvent plus s'en passer. La différence entre deux sports devient claire: II y a le skl de fond et le ski alpin (Laurin, 1989).

Johannsen poursuit la promotion du skI de fond toute sa vle. En 1932, Il commence à tracer la plste Maple Leaf entre Shawbridge et Sainte-Agathe-des-Monts, En 1939, 1600 kllomètres de pistes sont tracés entre les vlllages. On trouve aulourd'hul la Gillesple, la Johannsen, la Western. Cette même année pourtant, le premler téléstège canadien au mont Tremblant fait trồner définitivement le skl-rol: le skl alpin. Ce sport déclenche la deuxtème impulsion réussle dans Ie développement Saint-Jovite mont Tremblant. Les Américains sont encore de la partle à travers cette activité si prisée malgré son coût élevé. Le proprlétalre du Gray Rocks Inn, Whecler, falt venir d'Allemagne, en 1932, le moniteur BIII Pauly qui lance icl sa technique: le Berlin Squatt. SIx ans plus tard, il grossit son équipe d'instructeurs avec deux Tyrollens blen connus. Hans Faulkner et Herman Gardner. Pour la petite histoire: Gardner entraine Lucllle, la petite fille de Wheeler qul devlent, en 1958, la premlère Canadienne à gagner une médaille d'or en compétition Internationale. Ouand un Autrichien entraîne une fille d'Américains sur les pentes des Laurentides aux noms francophones!

\section{Mont Tremblant Lodge}

Mais l'apport entrepreneurial américain n'en reste pas là. Un habitant de Phlladelphle, Joseph Ryan, en février 1938. escalade le mont Tremblant avec ses amis Lowell Thomas et Harry Wheeler. Nouveau curé Labelle, II *volt* I'Immense développement touristique possible sur place pour le ski. Il appelle aussitôt l'interventlon en capital de son aml financier de Wall Strect, Ben Smith. Ryan va hésiter après avoir ouvert un chantier immense sur un territolre public... Le curé Hector Deslauriers, qui sait dans quelle crise économique se débattent ses paroissiens, intervient avec des pétltions et relance le premier ministre Maurice Duplessis qul crolt déjả au projet, pour qu'll vende ou loue des terres publiques à Ryan. La vellle de la deuxtème Guerre Mondiale, au coeur des Laurentides, fruit de la collaboration entre un financier 
et un entrepreneur étrangers, avec l'Etat et une parolsse, se bâtit un des plus gros centres de ski d'Amérique du Nord: le Mont Tremblant Lodge, avec son petit vlllage très couleur locale. Le prestlgleux Tlmes lul consacre mëme un article. Dès le premler hiver, l'élite d'affalres américaine en falt un de ses lleux de rencontre internationaux. On sait dans les années 1990, I'Investissement énorme falt à Mont-Tremblant. par Intra West qul défle toute concurrence au Québec et sans doute au Canada.

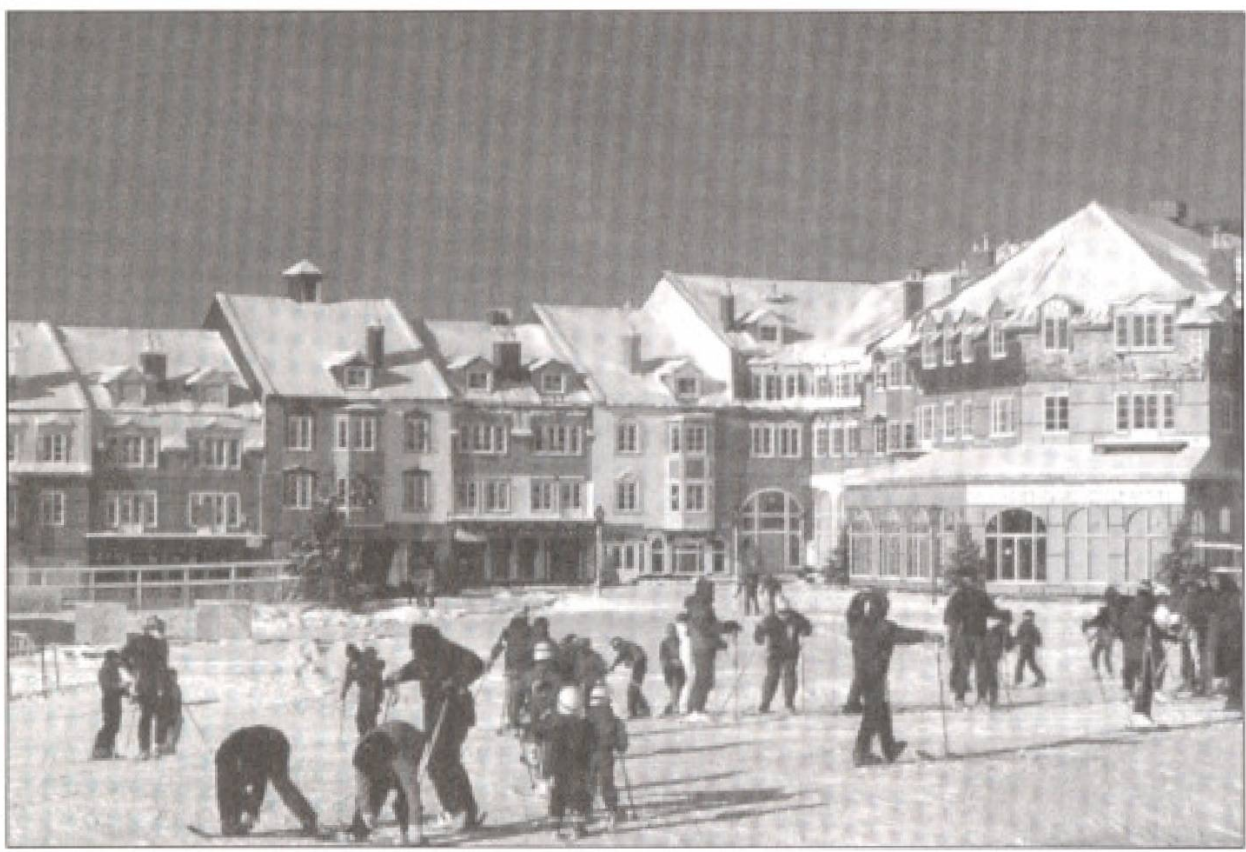

vent étranger. La villéglature, autre face du tourlsme dans les Laurentides, participe de l'apport ethnique. Il existe presque des clichés sur certains mouvements comme la place précoce des Julfs dans le développement de SainteAgathe-des-Monts: leur présence est restée aussl actlve. Poser la question: pourquol des Juifs à Salnt-Agathe?, c'est attendre à peu près la même réponse qu'à la questıon: pourquol des Russes a Rawdon? C'est souvent le hasard d'une Installation, la reconnalssance du lleu, les réminlscences paysagiques, la diffusion par le bouche à orelle, l'instinct grégaire du groupe, et alnsi de sulte.

Les Julfs qu'on se représente, au Québec, comme l'ethnie la plus urbaine, se rendent nombreux dans les Laurentides montréalalses. Raoul Blanchard, dans les années 1940. compte 400 familles julves à Prévost. Ils sont aussl à Pledmont avec des Ontariens et des Américains. A ValMorin, à Val-David les *coltages, chalets, débordent chaque été d'hôtes Israélltes:. Il en dénombre aussl à Saint-Faustin et blen sûr à SalnteAgathe-des-Monts. Aux IImItes de Lanaudière. Blanchard avance le chlffre de 250 familles lulves de Montréal, à Salnte-Sophle-deLacorne, venus rejolndre keurs corellglonalres flxés dans celte localltéw.

La plus grande singularité ethnıque. touristiquement parlant, est celle des

Les Américalns skleurs déferlent pendant des années dans les Pays-d'en-Haut, aussi bien à Mont-Tremblant, presque chez eux, qu'à Salnte-Marguerite, à l'Alpine Inn, entre autres. Ces mèmes américains vont |usqu'à surnommer *The Alouette Belt leur zone d"actlvité, en marlant leur catégorlsation à notre folklore! Dans Lanaudière. les Américains sont plutôt chasseurs et pêcheurs, clients des clubs comme Mastigouche. Des membres de la famille Kennedy organisent des expéditions de pêche au delà de Saint-Michel-des-Saints.

\section{Sainte-Marguerite-du-lac-Masson}

Un autre étranger allait donner un gros coup de pouce à Sainte-Maguerite-du-lac-Masson, un centre pourtant déjà appréclé. Il en fait une entreprise au moins aussi décisive pour ce vlllage que Ryan le fait trois ans plus tard pour son village adoptif. Le baron Louis Empain constrult le domaine de l'Estérel en 1936. II est le dernier flls de la famllle belge Empaln-Schnelder. Dès 1934, Il fonde des compagnies financlères axées sur le développement des Laurentıdes: crédit. immobilier, agriculture. L'hôtel de l'Estérel, dans un style architectural d'avant-garde, inauguré avec la musique de Benny Goodman, falt travalller les chômeurs des environs el devient une des perles de l'hébergement du Nord.

\section{La villégiature ethnique}

Liorganisation du skı alpln et de ses centres hôtellers est spectaculaire. Elle nécessite un lourd Investlssement, sou-
Russes, Lanaudlère abrite, dans les limites de Rawdon, une véritable oasls russe de villéglature et de résldence. Tout commence en 1929 quand un dame Kaghinskl ouvre une malson de pension. La réputation se répand d'un paysage de plnèdes à rendre nostalgique le molns sensible des Russes... De villéglateurs, de nombreux Russes sont devenus résldants. Plus de 50 famllles sont installées, dans les années 1990. Le nombre des villégiateurs est plus difficlle à évaluer. A Rawdon, vont aussi s'installer en salson et en permanence des étrangers en provenance d'autres pays de l'Europe de I'Est: des Polonals, des Hongrols, des Ukrainlens.

Cependant, si on excepte Rawdon, les Laurentides lanaudolses, n'ont pas attiré beaucoup les représentants des ethnies. Les deux réglons ont évidemment des villéglateurs non francophones que nulle statistique ne dénombre. II faudralt faire un relevé des taxes municipales de chaque munıcipalité et repérer tout nom à particularité étrangère... Les données du ministère présentent globalement les étrangers qul passent les frontlères pour activité touristique. Néanmoins, celul qul connaît empirıquement les territoires municipaux de villéglature sait que chaque village ou presque a sa colonle *d'étrangersm.

Alnsl Rawdon, dans les années 1930, après la perte de sa scierie, s'est mis au tourisme. Les chutes d'eau sont attlrantes. On bâtıt un lac artiflclel avec une plage. L'endrolt attire, en pleine crise, auss! bien les francophones que les 
anglophones. La villéglature anglophone la plus anclenne dans le pledmont de Lanaudière dolt être celle du principal de I'Université Mc Gill qui avalt en 1862 une résidence d'été à Saint-Gabrlel-de-Brandon. Cette localité devlendra, à partir des années 1930, un centre estival et hivernal très couru jusque dans les années 1960, mais beaucoup plus par les francophones.

\section{Une unité biophysique, mais deux régions touristiques.}

On peut alnsi, en conclusion, distinguer pour les Laurentides de l'Ouest (par opposition, comme le fait Blanchard, à celles de l'Est. de Québec à Maskinongé), deux zones ou deux Laurentides, qul correspondent aux deux réglons administrauves, pour ce qul est du tourisme ethnlque. On peut le falre également pour le tourlsme indigène. II existe simplement deux modèles de développement touristique, quil fondent en partle leur identité, expllqués par la proximité de Montréal, les voles de communication, =les rellefs à skl;, les Investlssements étrangers, la composition soclale des touristes (classes populaires et bourgeolsle), la notorlété précoce, les leaders d'opinlon, l'imagerle. Tous ces traits sont liés dialectlquement, On peut contunuer à parler d'une unité géotouristlque: les Laurentldes, mals II existe une franche réglonalisathon quil permet, à chacun des territolres de penser et de faire le tourisme différemment.

\section{Bibliographie}

Beauchamp-Forget, Jacques, Le skl dans les Laurentldes, premlers et premlères: chronologle Illustrée (1905-1982). Saint-Sauveur-des-Monts, Groupe de recherche sur l'histolre du ski dans les Laurentıdes, 1983.

Blanchard, Raoul. L'Ouest du Canada françals. Tome I. Montréal et sa réglon. Montréal, Beauchemin,1953.

Le centre du Canada françals. Montréal, Beauchemin,1947.

Brière, Roger, Les Laurentides montréalalses, pays de tourlsme, Bulletin de l'Assoclation des géographes de l'Amérique françalse, I1:97-106, 1967.

Filion. Mario , Une histoire des Pays -d'en-Haut. s.l., Société d'histolre des Pays- d'en-Haut, et Circa, 1981.

Fournler, Marcel, Rawdon: 175 ans d'Histolre. Jollette, s.e., 1974.

Katz, Elllot, Golden era of Laurentla skilng. Canadian Geographic, vol. 97, déc. 1978. Janvler 1979.

Laurin, Serge, Histoire des Laurentides, Québec, Institut québécols de la recherche sur la culture, 1989.

Lundgren. Yan, Le développement des entreprises d'hôtellerle dans les Laurentides montréalalses entre 1924 et 1965. Bulletin de l'Association des géographes de l'Amérlque françalse, 1I:113-121, 1967.

Morissonneau, Chrlstian, Gulde de Lanaudière. Jollette, Consell de la Culture de Lanaudière, 1985.

O'Rear, John, The Mont Tremblant Story Including Skilng the Mont Tremblant Way. New York, Barnes, 1954.

Saint-Amour, Jean-Plerre, La vlliéglature au Québec. Problématique de l'aménagement du territoire. Hull, Édltions Astico. 1979.

Soucy, Danlèle, La vallée de la Dlable: de la hache aux canons à nelge. Saint-Jovite. s.e, 1983

Vau, Robert, Le tourisme dans les Laurentides. Université de Montréal, thèse de maittrise en géographle, 1957. 